\title{
Atomic Diffusion and Electric Conductivity of Gum Arabic/Graphite Composite
}

\author{
Bilquis Ibrahim Adham¹, H. M. El-Khair ${ }^{1,2}$ \\ ${ }^{1}$ Department of Physics, Faculty of Science and Technology, Al Neelain University, Khartoum, Sudan \\ ${ }^{2}$ Comittee on Radiation and Environmental Pollution Protect (CREPP), Department of Physics, College of Science, Al Imam \\ Mohammad Ibn Saud Islamic University (IMSIU), Riyadh, KSA \\ Email: hatimdirar@gmail.com
}

How to cite this paper: Adham, B.I. and El-Khair, H.M. (2018) Atomic Diffusion and Electric Conductivity of Gum Arabic/Graphite Composite. Journal of Materials Science and Chemical Engineering, 6, 91-96

https://doi.org/10.4236/msce.2018.67010

Received: May 22, 2018

Accepted: July 6, 2018

Published: July 9, 2018

Copyright $\odot 2018$ by authors and Scientific Research Publishing Inc. This work is licensed under the Creative Commons Attribution International License (CC BY 4.0).

http://creativecommons.org/licenses/by/4.0/

\begin{abstract}
In this work, we had adopted solid states reaction kinetic model to calculate diffusion coefficient of graphite Gr in Gum Arabic (GA) as the function of temperatures and concentration $D(t, C)$. From the calculations we have found that the diffusion coefficient $D$ increases with increasing temperature. At higher temperature the $\mathrm{Gr}$ atoms might get enough thermal energy to overcome the activation energy $(\mathrm{Ea})$ barrier and hence can easily be transported to a new atomic position. Diffusion coefficient of Gr at high concentrations had exceeded that at low concentrations by more than two to three orders of magnitude. Such increase can be attributed to the large number of jumps or jump frequency which found to be directly proportion to the $\mathrm{Gr}$ concentration. Electric conductivity, calculated by Nernst-Einstein equation, at high concentration Gr had exceeded that at lower concentration. The decrease in conductivity with decreasing Gr concentration might be attributed to the effective charges interactions, which lead to enhance the recombination of charge carriers.
\end{abstract}

\section{Keywords}

Graphite, Polymers, Composites, Temperature Dependence, Diffusion Mechanism

\section{Introduction}

Carbon had remained to be important for various applications due to its novel physical and chemical properties of its different isomers [1]. Carbon has said to be the most common element in nature and can be easily being obtained from any simple combustion of living and nonliving wastes [2]. Different thermodynamic conditions can lead to different types of carbon isomer. The physical 
properties of these isomers were found to be ranging from most plastic to most elastic carbon and from electrically insulator to a good conductor. Carbon-carbon and silicon-silicon bonds can reinforce the thermo-elastic properties of materials and hence make it capable for being used for material reinforcement [3]. Such a successful utilization of carbon had motivated scientific research and leads to the discovery of materials novel properties. Formation of three-dimensional metallic carbon [4], under ambient conditions, had showed the highest thermal and mechanical stability [5]. Electrical properties of polystyrene have been enhanced by filling it with carbon black [6] [7]. Enhance the structure, mechanical and electromagnetic properties of these new composite had been controlled by the thermodynamic conditions leading to the diffusion of carbon atoms in different polymeric matrix [8]. Thermal properties of lubricants have been improved by carbon nanophases [9]. Graphite as nano additives had improved the functionality of grease. Such nano-grease had acquired an excellent frictional property as well as modified anti-wear properties [10]. In this work, solid state reaction kinetic model was used to calculate diffusion coefficients and electric conductivity of Gr/GA composite as a function of temperatures and concentrations.

\section{Material and Method}

Gum Arabic and Graphite powder of high purities have been supplied by the Sudanese Gum Arabic and local Techno-Chemical local companies, respectively. Samples were prepared by solid state reaction by blending $1 \mathrm{~g}$ fine powder GA with $0.01,0.09$ and $0.25 \mathrm{~g}$ fine powder $\mathrm{Gr}$ forming, $\mathrm{S}_{1}, \mathrm{~S}_{2}$ and $\mathrm{S}_{3}$ respectively. The samples were subjected to thermal treatment at different temperatures (kelvin) ranging from 300,600 degree $\mathrm{K}$ at equal rate of 10 seconds.

\section{Background}

Let us consider any two elements A and B in solid phase and let A type atoms be a solute and $B$ type be a solvent atoms which are to be subjected mixing and heating. Due to such physical processing type A and B atoms may react to form composite. In our case the B-type atoms are GA whereas the A type are Gr. GA has said to be natural polymer of a complex chemical structure composed of different molecular groups of different chemical bonds. Which are highly affected by thermodynamic conditions.

Their atomic diffusion has been activated thermally. According to the thermodynamics the information concerning microstates can be traceable by the grand thermodynamic function includes macrostates variables such as volume, temperature, pressure etc. We will try to drive solid state reaction rate from the function of weight fraction calculated from the ratio between the mass difference before and after thermal treatment [10] [11]. The mass loss ratio $a$ can be calculated using the following equation

$$
\alpha=\frac{m_{0}-m_{T}}{m_{0}-m_{f}}
$$


where, $m_{0}$ and $m_{T}$ are the weights before and after thermal treatment, respectively whereas $m_{f}$ is the final weight obtained at the final thermal treatment stage [10] [11]. According to A. Khawan bintegral the functional relation between $\alpha$ and the kinetic parameter $k$, considering 3-D atomic diffusion is given as

$$
g(\alpha)=k t
$$

where,

$$
g(\alpha)=\left[1-(1-\alpha)^{\frac{1}{3}}\right]^{2}
$$

Hence,

$$
k=\frac{\left(1-(1-\alpha)^{\frac{1}{3}}\right)^{2}}{t}
$$

According to Arrhenius equation

$$
k=A \mathrm{e}^{\frac{-E_{a}}{R T}}
$$

where, $A, E_{a}$ and $R$ are the frequency factor, activation energy and gas constant, respectively. The activation energy and the frequency factor can be estimated from the linear plot relation between $\frac{1}{T} v s \cdot \ln (k)$. The activation energy calculated were used for determining the atomic diffusivity $(D)$ according to the equation

$$
D=D_{0} \mathrm{e}^{\frac{-E_{a}}{R T}}
$$

The direct relation between atomic diffusivity $(D)$ and electrical conductivity $(\sigma)$ is given by Nernst-Einstein equation

$$
\sigma=\frac{D N q^{2}}{K_{B} T}
$$

where, $\mathrm{N}_{\mathrm{A}}, q$ and $K_{B}$ are Avogadro's number, charge number and Boltzmann constant, respectively.

\section{Results and Discussion}

Diffusion coefficient of $\mathrm{Gr}$ had been calculated using solid state reaction kinetic model based on the temperature dependent mass variations [11]. Figure 1 shows the variation of diffusion coefficient with temperature and concentration. The diffusion coefficient at low concentration graphite had increased exponentially with increasing temperature. The diffusion coefficients of $S_{3}$ had exceeded that of $S_{2}$ and $S_{1}$ by 10 to 100 orders of magnitudes as shown in Figure 1. Such increase may be due to the high concentration of unconsumed vacancies created by the thermally activated $\mathrm{Gr}$ atoms. The density number of vacancies had been expected to increase with increasing temperature even in absence of dislocation. 


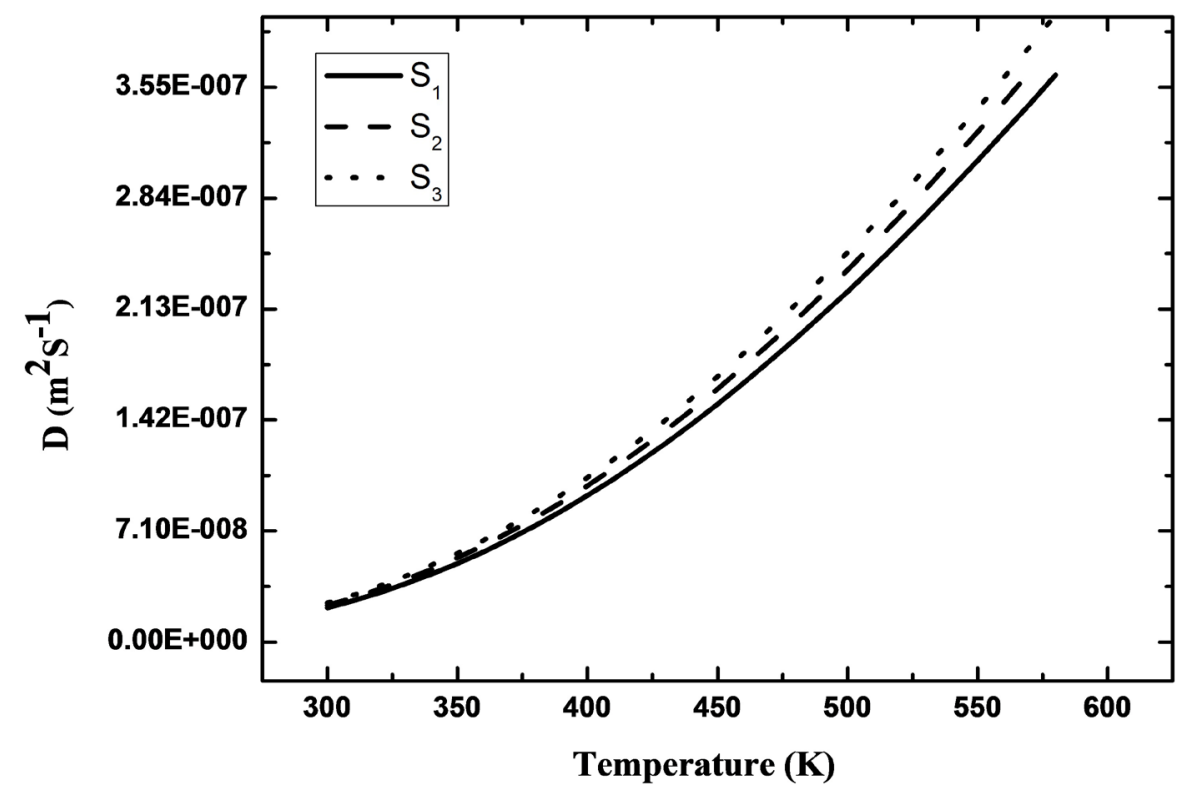

Figure 1. Shows variation of Diffusion coefficient with temperature.

Such vacancies represent high potential sites for diffusing Gr atoms in GA [12]. Significant shifts had been detected from the curves $S_{1}, S_{2}$ and $S_{3}$ as being depicted in Figure 1. These shifts can be attributed to the formation of vacancies with different density number and energies. Increase in $\mathrm{Gr}$ concentration might lead to the large number of random motion of atoms and hence more jumps from one atomic site to anther resulting in fast diffusion [13]. At higher temperature the $\mathrm{Gr}$ atoms might get enough thermal energy to overcome the activation energy (Ea) barrier and hence can easily be transported to a new atomic position. Diffusion coefficient of $\mathrm{Gr}$ at high concentrations had exceeded that at low concentrations by more than two to three orders of magnitude. Variation of the electrical conductivity, calculated by Nernst-Einstein Equation (7) with temperature has been depicted in Figure 2. From Figure 2 it is obviously seen that, the electric of conductivity $S_{3}$ exceeds that of $S_{1}$ and $S_{2} \cdot G A / G r$ composites has said to be highly disordered, the charge transport might be due to the quantum hopping which is to be supported by the interaction of thermal phonons with charge carriers and accordingly it moves from one site to another site of same or different energies [14] [15]. From the features of the curves we have seen that at low concentration $\mathrm{Gr}$ the interaction between charge carriers might be negligible and the temperature dependent electrical conductivity might follow exponential relation [15] of the form;

$$
\sigma(T)=\sigma_{0} \exp \left[\left(-\frac{T_{0}}{T}\right)^{\gamma}\right]
$$

where the exponent $\gamma$ is related to the dimensionality and $\gamma=\frac{1}{4}$.

The decrease in conductivity with decreasing Gr concentration might be attributed to the effective charges interactions, which lead to enhance the 


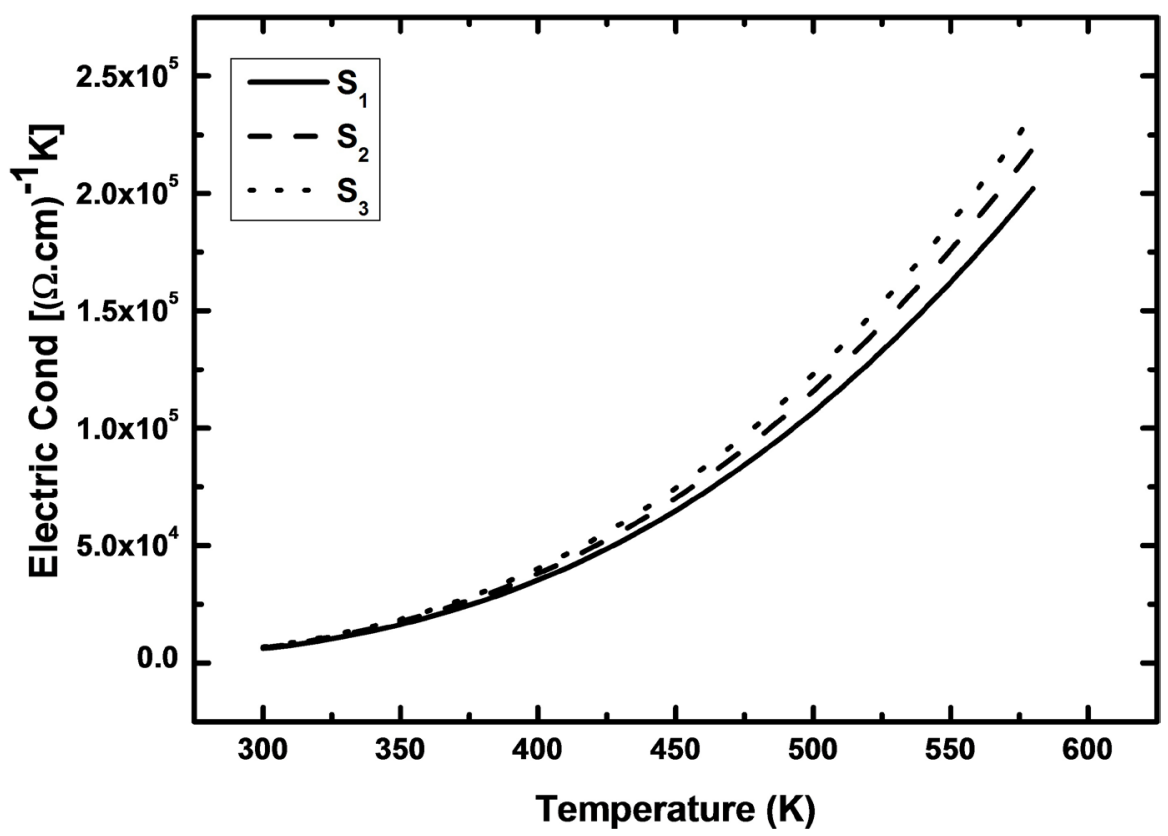

Figure 2. Shows the variation of electric conductivity with temperature.

recombination of charge carriers.

This work we had pinned pointed the effect of continuous thermal treatment on the Gr diffusion coefficients in GA and its effect in electrical conductivity of the $\mathrm{Ga} / \mathrm{Gr}$ composite. The results had showed increase in the diffusion coefficient with temperature at high Gr concentrations. The increase in Gr concentration might results in considerable charge carrier's interactions. It was clearly seen that, the electrical conductivity calculations indicate that the GA/Gr composite had turn into a semiconductor. Such processing had produced novel engineering material.

This work is supported by the Material Science Research Laboratory of Al-Neelain University and the Ministry of Higher Education and Scientific Research of the.

\section{References}

[1] Pierson, H.O. (2012) Handbook of Carbon, Graphite, Diamonds and Fullerenes: Processing, Properties and Applications. William Andrew, Amsterdam.

[2] Wall, T.F. (2007) Combustion Processes for Carbon Capture. Proceedings of the Combustion Institute, 31, 31-47. https://doi.org/10.1016/j.proci.2006.08.123

[3] Zakhidov, A.A., et al. (1998) Carbon Structures with Three-Dimensional Periodicity at Optical Wavelengths. Science, 282, 897-901. https://doi.org/10.1126/science.282.5390.897

[4] Okada, S., Susumu, S. and Atsushi, O. (1999) New Metallic Crystalline Carbon: Three Dimensionally Polymerized C 60 Fullerite. Physical Review Letters, 83, 1986. https://doi.org/10.1103/PhysRevLett.83.1986

[5] Du, J.H. and H, M.C. (2012) The Fabrication, Properties, and Uses of Graphene/Polymer Composites. Macromolecular Chemistry and Physics, 213, 1060-1077. https://doi.org/10.1002/macp.201200029 
[6] Zhou, X.-W., Zhu, Y.-F. and Liang, J. (2007) Preparation and Properties of Powder Styrene-Butadiene Rubber Composites Filled with Carbon Black and Carbon Nanotubes. Materials Research Bulletin, 42, 456-464. https://doi.org/10.1016/j.materresbull.2006.06.027

[7] Pant Krishna, P.V. and Boyd, R.H. (1993) Molecular-Dynamics Simulation of Diffusion of Small Penetrants in Polymers. Macromolecules, 26, 679-686. https://doi.org/10.1021/ma00056a019

[8] Sanes, J., et al. (2017) Synergy between Graphene and Ionic Liquid Lubricant Additives. Tribology International, 116, 371-382. https://doi.org/10.1016/j.triboint.2017.07.030

[9] Anand, G. and Prateek, S. (2016) A Review on Graphite and Hybrid Nano-Materials as Lubricant Additives. IOP Conference Series: Materials Science and Engineering, 149, No. 1. https://doi.org/10.1088/1757-899X/149/1/012201

[10] Šesták, J. and Berggren, G. (1971) Study of the Kinetics of the Mechanism of Solid-State Reactions at Increasing Temperatures. Thermochimica Acta, 3, 1-12. https://doi.org/10.1016/0040-6031(71)85051-7

[11] Miotello, A., et al. (2001) Clustering of Gold Atoms in Ion-Implanted Silica after Thermal Annealing in Different Atmospheres. Physical Review B, 63, 075409. https://doi.org/10.1103/PhysRevB.63.075409

[12] Wilcox, W.R. and LaChapelle, T.J. (1964) Mechanism of Gold Diffusion into Silicon. Journal of Applied Physics, 35, 240-246. https://doi.org/10.1063/1.1713077

[13] Kwok, T., Ho, P.S. and Yip, S. (1984) Molecular-Dynamics Studies of Grain-Boundary Diffusion. I. Structural Properties and Mobility of Point Defects. Physical Review B, 29, 5354. https://doi.org/10.1103/PhysRevB.29.5354

[14] Sheng, P., Abeles, B. and Arie, Y. (1973) Hopping Conductivity in Granular Metals. Physical Review Letters, 31, 44. https://doi.org/10.1103/PhysRevLett.31.44

[15] Marschall, R. (2014) Semiconductor Composites: Strategies for Enhancing Charge Carrier Separation to Improve Photo Catalytic Activity. Advanced Functional Materials, 24, 2421-2440. https://doi.org/10.1002/adfm.201303214 